\title{
Nonlinear visco-elastic and accelerating creep model for coal under conventional triaxial compression
}

\author{
Sheng-Qi Yang $\cdot$ Peng Xu • Tao Xu
}

Received: 2 July 2015/Accepted: 29 October 2015/Published online: 4 November 2015

(C) Springer International Publishing Switzerland 2015

\begin{abstract}
On basis of triaxial creep experimental results of coal with various deviatoric stress levels, a new nonlinear visco-elastic and accelerating creep model is put forward. First, Burgers visco-elastic triaxial creep model is deduced and used to identify the creep parameters by the discrimination criterion of the square of correlation coefficient $\left(R^{2}\right)$ and sum of least error square $(Q)$. In Burgers visco-elastic triaxial creep model, the visco-elastic modulus $G_{2}$ of coal decreases but the visco coefficient $\eta_{1}$ and $\eta_{2}$ all increase with increasing time, which has distinct time scale effect. The Burgers visco-elastic triaxial creep model fails in predicting long-term deformation characteristics. Then by introducing a time index, a nonlinear visco-elastic triaxial creep model is put forward, which can predict better for long-term deformation behavior of coal. $R^{2}$ identified by nonlinear visco-elastic triaxial creep model is higher, but $Q$ identified by nonlinear visco-elastic triaxial creep model is smaller 1 order than that by Burgers triaxial
\end{abstract}

S.-Q. Yang $(\bowtie) \cdot$ P. Xu

State Key Laboratory for Geomechanics and Deep Underground Engineering, School of Mechanics and Civil Engineering, China University of Mining and Technology, Xuzhou 221116, People's Republic of China e-mail: yangsqi@hotmail.com

T. $\mathrm{Xu}$

Center for Rock Instability and Seismicity Research, Northeastern University, Shenyang 110819, People's Republic of China creep model. However, Burgers and nonlinear viscoelastic triaxial creep model is very difficult to describe the accelerating creep stage of coal. Therefore in the end, a new nonlinear accelerating creep model is put forward in order to describe the complete creep curve of coal. The nonlinear accelerating creep model has a better agreement with the experimental data.

Keywords Coal - Triaxial compression · Nonlinear · Visco-elastic triaxial creep model · Accelerating creep model

\section{Introduction}

Coal is a kind of special rock material containing many minor microscopic fissures. In the past, a lot of short-term strength, deformation and acoustic emission (AE) behaviors of coal have been experimentally investigated (Medhurst and Brown 1998; Feng et al. 2010; Li et al. 2011; Xie et al. 2012). However, very fewer test results have been reported regarding creep behaviors of coal under uniaxial and conventional triaxial compression. Cao et al. (2007) carried out the experiment on outburst-hazardous coal under uniaxial compression creep to study the $\mathrm{AE}$ characteristics of coal at different creep stages. But the creep time of coal was too short, only $4 \mathrm{~h}$. Wang et al. (2010) used self-creep testing apparatus to carry out triaxial creep tests on outburst coal specimens from Songzao coal mine, which found that the attenuation creep property 
was shown in the conditions of less than its long-term strength load, which non-attenuation creep property for the greater conditions. Yin et al. (2011) carried out the triaxial creep experiment of coal containing gas under unloading condition. The results showed that unloading could accelerate the failure and the macrofissure induced by unloading could increase the gas flow velocity significantly. Xu et al. (2009) analyzed the influence of creep on permeability of gasbearing coal by means of experimental equipments of coal seepage. The experimental results showed that with different temperatures and effective stress coupling, the influence of creep on coal permeability was also different. The above creep test results of coal build a better foundation for the construction of creep models of coal.

In recent years, a few progresses on triaxial creep model of coal have been reported. Based on triaxial creep test results of out burst prone coal, by introducing a visco-elasto-plastic body which could describe the deformation property of non-Newtonian fluids, Wang et al. (2010) put forward the viscoelasto-plastic creep model of out burst prone coal combined with a Poyting-Thomson model. On the basis of the triaxial creep experimental data of coal containing gas, Yin et al. (2011) modified Chaboche viscoplasticity constitutive relation, which could describe the short term creep failure of coal containing gas. However, the previous triaxial creep models do not take into account time scale effect and verify the predicted effect of triaxial creep model with various differential stress levels, therefore which can not be used to predict long-term creep deformation of coal. Up to now, the theories of triaxial creep model of coal materials have not been very mature. Nowadays, triaxial creep model of coal is still one of the most difficult problems in the rock rheological mechanics study.

Therefore in this research, to better understand triaxial creep behavior and predict long-term deformation behavior of deep coal tunnel, triaxial creep tests were conducted for coal located in Xingan mine of China with various axial differential stress levels. Based on visco-elastic triaxial creep test results of coal, the variance of creep parameters with time were analyzed in detail. The paper focuses on modeling of triaxial creep deformation by putting forward nonlinear visco-elastic and accelerating creep model for coal under conventional triaxial compression, and evaluating the accuracy and reasonability of identified triaxial creep parameters.

\section{Triaxial creep test result of coal}

The coal located in the deep coal tunnel (at a depth of about $700 \mathrm{~m}$ ) of Xingan mine was chosen for the test object. Coal blocks were collected from in-site engineering and then were further machined in the laboratory. We made cylindrical coal specimen with the length of $100 \mathrm{~mm}$ and the diameter of $50 \mathrm{~mm}$ according to ASTM requirement (1996) for creep testing. The coal specimens have no obvious fractures and cracks, but exist some minor microscopic fissures in accordance with the scanning electric microscopic (SEM) results (Fig. 1). At the same time, coal intrinsically has anisotropy due to its cleat structure; therefore, we machined the coal specimens along the same direction from the same block in order to avoid the influence of anisotropy on the creep experimental results of coal.

We carried out the triaxial creep tests for coal specimen on a rock servo-controlled triaxial rheology testing system. The testing system is made up of a loading system, constant-stability pressure equipment, a hydraulic pressure transferring system, pressure chamber equipment, a water pressure system and an automatic data collection system. This testing system can be used to perform hydrostatic pressure tests, conventional triaxial compression tests under drained or undrained conditions, triaxial seepage tests, triaxial creep tests and chemical corrosion tests.

The triaxial creep tests were conducted in the laboratory with constant temperature and humidity to avoid the influence of the disturbance of surrounding environment on the experimental results. During the triaxial compression tests, the confining pressure was firstly increased to the pre-confirmed value at a rate of $0.5 \mathrm{MPa} / \mathrm{s}$ in order to ensure coal specimen was in a uniform hydrostatic stress. Then the axial differential stress was applied on the rock specimens at a constant rate of $0.127 \mathrm{MPa} / \mathrm{s}$. In accordance with short-term triaxial strength of coal, the axial differential stress level of triaxial creep test can be determined. The confining pressure is set to $30 \mathrm{MPa}$, and the corresponding short-term triaxial strength of coal is about 82.43 MPa, denoting with the axial differential stress. Therefore two axial differential stress levels, i.e. 73 

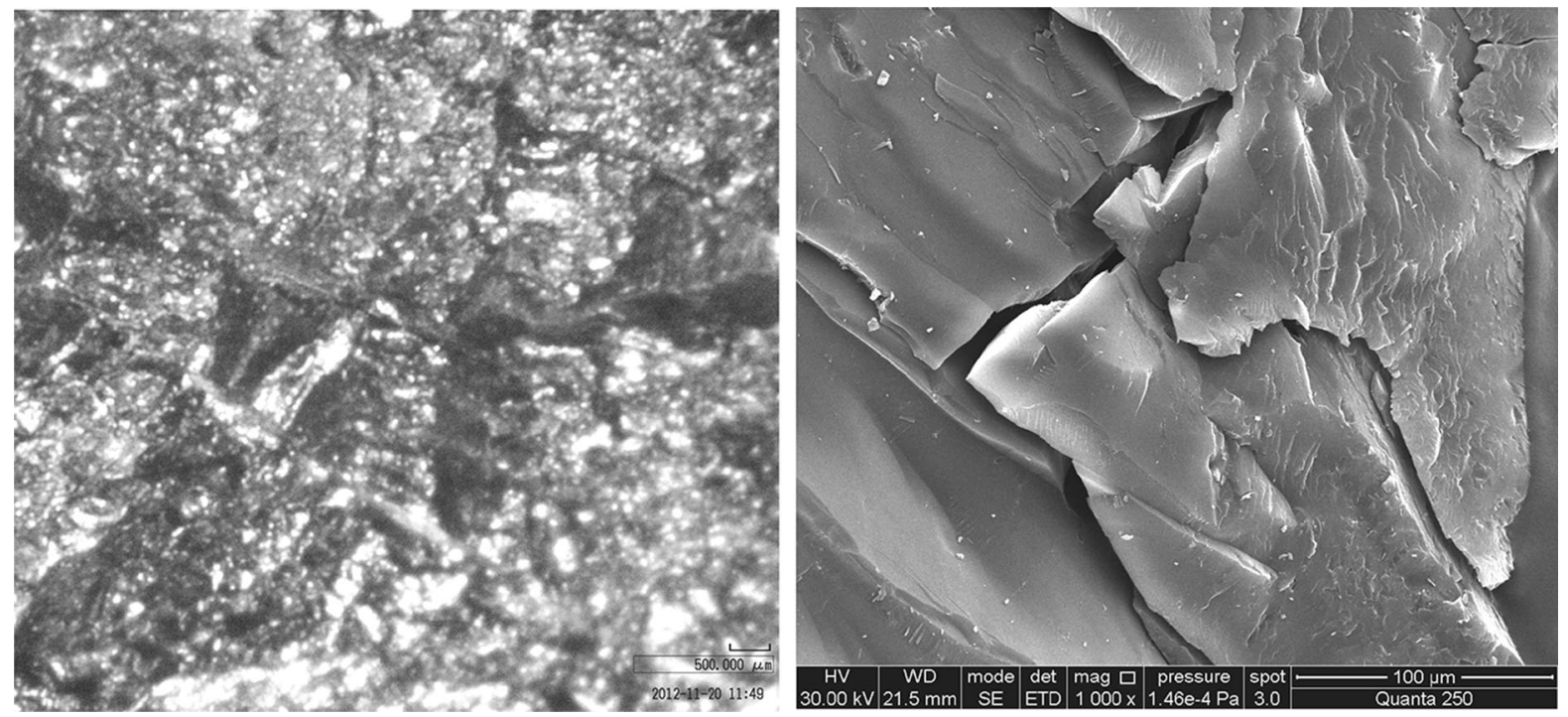

Fig. 1 Microscopic observation of tested coal specimen in the present specimen

and $75 \mathrm{MPa}$, were used to investigate the triaxial creep behavior of coal. When testing, at first, a constant confining pressure $(30 \mathrm{MPa})$ was loaded on coal specimen, and then applied the corresponding axial differential stress level. The relation between axial strain and time were obtained at varying axial differential stress levels.

Figure 2a shows obtained typical visco-elastic triaxial creep test results of coal at constant axial differential stress level. In terms of Fig. 2a, instant deformation occurs in the coal at constant confining pressure. With the increase of time, the axial strain of coal specimen also increases step by step, but axial creep rate decreases gradually. After the time increases to $50 \mathrm{~h}$ (about 2 days), the coal specimen transforms from primary creep stage to steady-state creep stage, and the axial deformation increases linearly with time by a constant steady-state creep rate.

It should be noted that the coal specimen does not occur the creep failure at the axial differential stress of $73 \mathrm{MPa}$ and only two creep stages (i.e. primary creep stage and steady-state creep stage) occurs. However, when the axial differential stress level is increased to $75 \mathrm{MPa}$, the creep failure occurs as illustrated in Fig. 2b, which shows a different creep behavior compared with that at the axial differential stress of $73 \mathrm{MPa}$.

From Fig. 2b, it can be seen that when the confining pressure equals to $30 \mathrm{MPa}$ and the axial differential stress reaches $75 \mathrm{MPa}$, the axial deformation of coal specimen undergoes three stages of primary creep (AB), steady-state creep (BC) and accelerating creep (CD) and corresponds to primary creep rate, steadystate creep rate and accelerating creep rate, respectively in the axial direction. At primary creep stage, axial creep strain rate decreases to a constant value quickly with the increase of time. At steady-state creep stage, axial creep strain rate keeps basically a constant with the increase of time. At accelerating creep stage, axial creep strain rate increases sharply with the increase of time and the creep failure occurs in coal specimen.

\section{Burgers visco-elastic triaxial creep model and time scale effect}

Based on triaxial creep curves of coal in Fig. 2a, it can be seen that the coal has distinct visco-elastic behavior with a steady-state creep rate higher than zero. To describe visco-elastic triaxial creep behavior of coal, two basic components, i.e. an elastic component (Hookean body) and a visco-elastic component (Newton body), are often connected in series or parallel, which can form all kinds of visco-elastic creep models, such as Maxwell model (Hookean body and Newton body are connected in series), Kelvin model (Hooker body and Newton body are connected in parallel). In this paper, the Burgers visco-elastic model 
Fig. 2 Typical viscoelastic and accelerating triaxial creep test curves of coal specimens at different deviatoric stresses
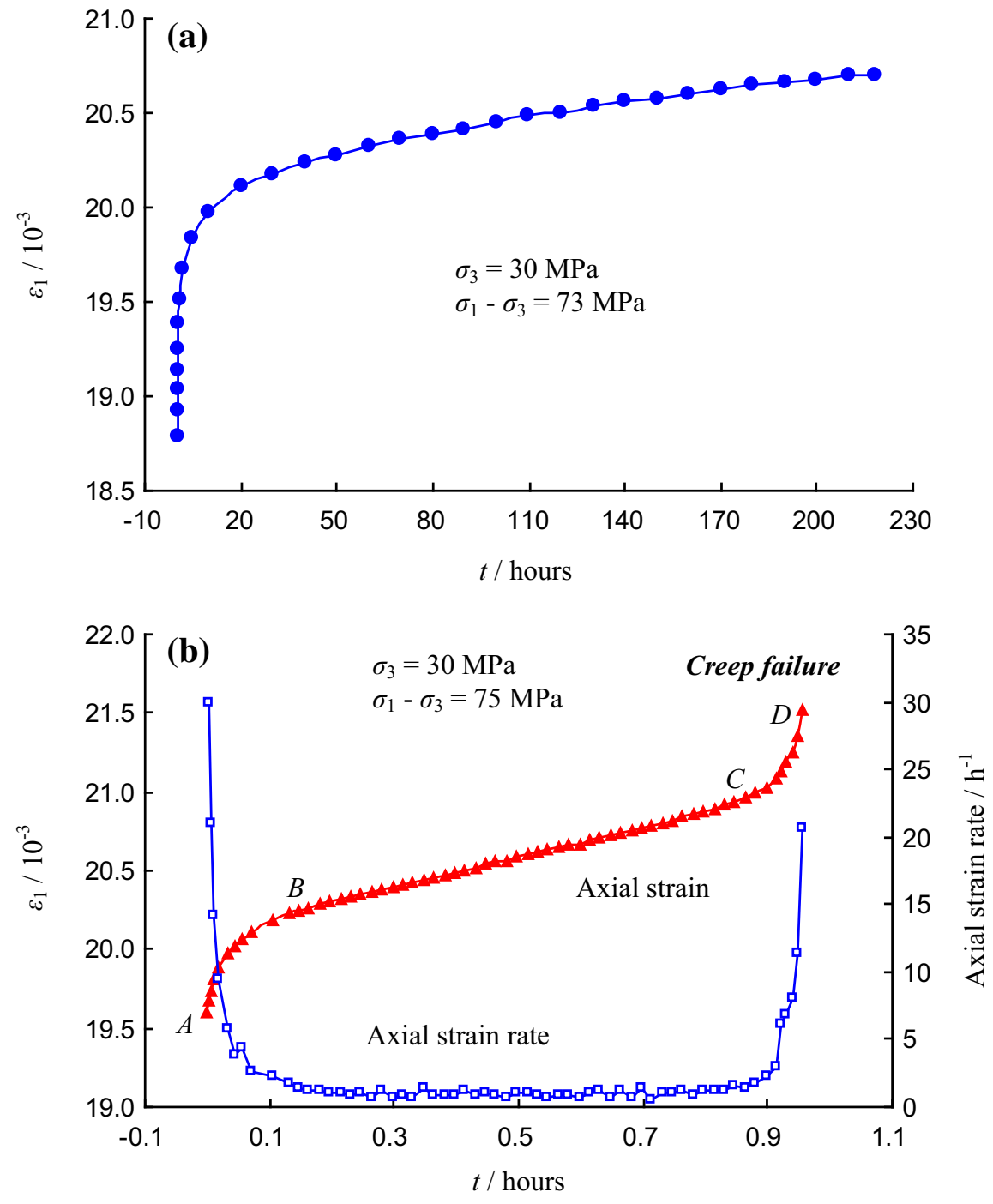

is chosen to study the visco-elastic triaxial creep behavior of coal. The creep equation of Burgers model under one-dimensional stress state (i.e. uniaxial compression state) is expressed as

$\varepsilon_{1}=\frac{\sigma}{E_{1}}+\frac{\sigma}{E_{2}}\left[1-\exp \left(-\frac{E_{2}}{\eta_{1}} t\right)\right]+\frac{\sigma}{\eta_{2}} t$

where $E_{1}$ is the elastic modulus; $E_{2}$ is the visco-elastic modulus; $\eta_{1}$ and $\eta_{2}$ are all the visco coefficient.

However, engineering rock mass is usually located in a triaxial compressive stress state. Therefore, we will deduce further the creep equation of rock under three-dimensional stress state based on the above mentioned creep equation under one-dimension stress state (Bonini et al. 2009).
When rock is in a three-dimension stress state, its internal stress tensor may be decomposed into a spherical stress tensor $\sigma_{m}$ and a differential stress tensor $S_{i j}$,

$$
\left\{\begin{array}{l}
\sigma_{m}=\frac{1}{3}\left(\sigma_{1}+\sigma_{2}+\sigma_{3}\right)=\frac{1}{3} \sigma_{k k} \\
S_{i j}=\sigma_{i j}-\delta_{i j} \sigma_{m}=\sigma_{i j}-\frac{1}{3} \delta_{i j} \sigma_{k k}
\end{array}\right.
$$

where, $\delta_{\mathrm{ij}}$ is Kronecker function. Thus, in accordance with Eq. (3), the following equation holds

$\sigma_{i j}=S_{i j}+\delta_{i j} \sigma_{m}$

The spherical tensor $\sigma_{m}$ can only change the volume of object, but not its shape. On the other hand, the 
differential stress tensor $S_{i j}$ can only change the shape of object, but not its volume. Therefore, strain tensor can also be decomposed into a spherical strain tensor $\varepsilon_{m}$ and a differential strain tensor $e_{i j}$,

$$
\left\{\begin{array}{l}
\varepsilon_{m}=\frac{1}{3}\left(\varepsilon_{1}+\varepsilon_{2}+\varepsilon_{3}\right)=\frac{1}{3} \varepsilon_{k k} \\
e_{i j}=\varepsilon_{i j}-\delta_{i j} \varepsilon_{m}=\varepsilon_{i j}-\frac{1}{3} \delta_{i j} \varepsilon_{k k}
\end{array}\right.
$$

Therefore, the following equation can also be obtained.

$\varepsilon_{i j}=e_{i j}+\delta_{i j} \varepsilon_{m}$

Suppose the shear modulus of rock is $G$, and bulk modulus of rock is $K$, the following relation relates these two properties

$$
\begin{aligned}
& G=\frac{E}{2(1+\mu)} \\
& K=\frac{E}{3(1-2 \mu)}
\end{aligned}
$$

where, $E$ and $\mu$ are, respectively, the elastic modulus and Poisson's ratio of the rock material.

For the Hookean Body under a three-dimension stress state, we have

$$
\begin{gathered}
\sigma_{m}=3 K \varepsilon_{m} \\
S_{i j}=2 G e_{i j}
\end{gathered}
$$

For a visco-elastic body under three-dimension stress state, its creep equation can be derived in accordance with the creep equation under uniaxial stress state, the constant differential stress of rock, written as

$$
\left\{\begin{array}{l}
\varepsilon_{m}=\frac{1}{3 K} \sigma_{m} \\
e_{i j}=\frac{S_{i j}}{2 G_{1}}+\frac{S_{i j}}{2 G_{2}}\left[1-\exp \left(-\frac{G_{2}}{\eta_{1}} t\right)\right]+\frac{S_{i j}}{2 \eta_{2}} t
\end{array}\right.
$$

Therefore, the creep equation of rock under conventional triaxial compression stress condition $\left(\sigma_{2}=\sigma_{3}\right)$ can be written as

$$
\begin{aligned}
\varepsilon_{1}= & \frac{\sigma_{1}+2 \sigma_{3}}{9 K}+\frac{\sigma_{1}-\sigma_{3}}{3 G_{1}} \\
& +\frac{\sigma_{1}-\sigma_{3}}{3 G_{2}}\left[1-\exp \left(-\frac{G_{2}}{\eta_{1}} t\right)\right]+\frac{\sigma_{1}-\sigma_{3}}{3 \eta_{2}} t
\end{aligned}
$$

In accordance with triaxial creep test curves, the square of correlation coefficient $\left(R^{2}\right)$ and sum of least error square $(Q)$ are simultaneously chosen as discrimination criterion to evaluate the creep model parameters obtained by nonlinear least square method (NLSM). It needs to be noted that $R^{2}$ more tends to 1.0 and $Q$ more approaches to zero, which means that the theoretical model agrees better with the experimental data. Therefore for a given creep model and experimental data, we always can obtain a maximum $R^{2}$ and a minimum $Q$.

The creep parameters in Eq. (11) for Burgers model can be identified by the following method. First, the bulk modulus $K$ and shear modulus $G_{1}$ can be obtained by $\varepsilon_{0}$, where $\varepsilon_{0}$ is instant axial strain for each axial differential stress level. Take the axial differential stress level $\sigma_{1}-\sigma_{3}=73 \mathrm{MPa}$ as an example, $\varepsilon_{0}$ is equal to $18.782 \times 10^{-3}$. Thus, we can obtain the following equation,

$\varepsilon_{0}=\frac{\sigma_{1}+2 \sigma_{3}}{9 K}+\frac{\sigma_{1}-\sigma_{3}}{3 G_{1}}=18.782 \times 10^{-3}$

In accordance with Eq. (12) and Eqs. (6-7), we can obtain the bulk modulus $K$ and shear modulus $G_{1}$ of coal are respectively 4.131 and $1.690 \mathrm{GPa}$. After confirming the parameters $K$ and $G_{1}$, other three creep parameters $G_{2}, \eta_{1}$ and $\eta_{2}$ can be obtained by direct iterative method using NLSM. According to $m$ groups of experimental data $\left(t_{\mathrm{i}}, \varepsilon_{i}\right)$ ( $\mathrm{i}=1, \ldots, m)$, a group of initial approximate values $\left(G_{2}^{0}, \eta_{1}^{0}\right.$ and $\left.\eta_{2}^{0}\right)$ is firstly given, and then many iterations are carried out on basis of initial approximate values until the creep parameters satisfies required precision by $R^{2}$ and $Q$ as discrimination criterion. When $R^{2}$ and $Q$, respectively, reach a maximum and minimum value, the corresponding creep parameter values are that we need.

By adopting the above method, the creep model parameters with eight various time scales (i.e. $t=20$, $50,80,110,140,170,200$ and $218 \mathrm{~h}$ ) are identified to analyze the influence of long-and-short of creep test time on Burgers creep model parameters. Table 1 lists the Burgers visco-elastic triaxial creep model parameters of coal with different time scales. A maximum $R^{2}$ and a minimum $Q$ are also obtained. Notice, $K$ and $G_{1}$ are constant for a given axial differential stress level, which are independent on time scale. However, the creep parameters $G_{2}, \eta_{1}$ and $\eta_{2}$ change with the increase of time scale, as shown Fig. 3. From Fig. 3, 
Table 1 Burgers visco-elastic creep model parameters of coal with axial differential stress level of $73 \mathrm{MPa}\left(\sigma_{3}=30 \mathrm{MPa}\right)$

\begin{tabular}{lllllrrr}
\hline Time scale $(\mathrm{h})$ & $K(\mathrm{GPa})$ & $G_{1}(\mathrm{GPa})$ & $G_{2}(\mathrm{GPa})$ & $\eta_{1}(\mathrm{GPa} h)$ & $\eta_{2}(\mathrm{GPa} \mathrm{h})$ & $R^{2}$ & $Q$ \\
\hline 20 & 4.131 & 1.690 & 29.24 & 6.98 & 882.15 & 0.974 & $6.38 \times 10^{-6}$ \\
50 & 4.131 & 1.690 & 25.22 & 8.71 & 1967.29 & 0.970 & $9.50 \times 10^{-6}$ \\
80 & 4.131 & 1.690 & 23.35 & 9.86 & 2945.65 & 0.970 & $1.065 \times 10^{-5}$ \\
110 & 4.131 & 1.690 & 22.14 & 10.79 & 3862.63 & 0.970 & $1.114 \times 10^{-5}$ \\
140 & 4.131 & 1.690 & 21.28 & 11.59 & 4696.98 & 0.970 & $1.126 \times 10^{-5}$ \\
170 & 4.131 & 1.690 & 20.61 & 12.31 & 5474.32 & 0.970 & $1.130 \times 10^{-5}$ \\
200 & 4.131 & 1.690 & 20.05 & 13.00 & 6211.93 & 0.970 & $1.133 \times 10^{-5}$ \\
218 & 4.131 & 1.690 & 19.73 & 13.45 & 6700.88 & 0.970 & $1.142 \times 10^{-5}$ \\
Average & 4.131 & 1.690 & 22.70 & 10.84 & 4092.73 & 0.971 & $1.037 \times 10^{-5}$ \\
\hline
\end{tabular}

we can see that with the increase in time, the viscoelastic shear modulus $G_{2}$ of coal decrease step by step and the variance tends to slow down. But it can be seen that the visco coefficient $\eta_{1}$ of coal increases step by step and the variance tends to slow down. However, it can be seen that the visco coefficient $\eta_{2}$ of coal increase with time, which has almost a linear behavior.
From the above analysis, we conclude that Burgers visco-elastic triaxial creep model parameters have obvious time scale effect, i.e. identified visco-elastic triaxial creep model parameters of coal are distinctly different with various time scales. Therefore, the Burgers visco-elastic triaxial creep parameters identified by short-term creep test results are very difficult
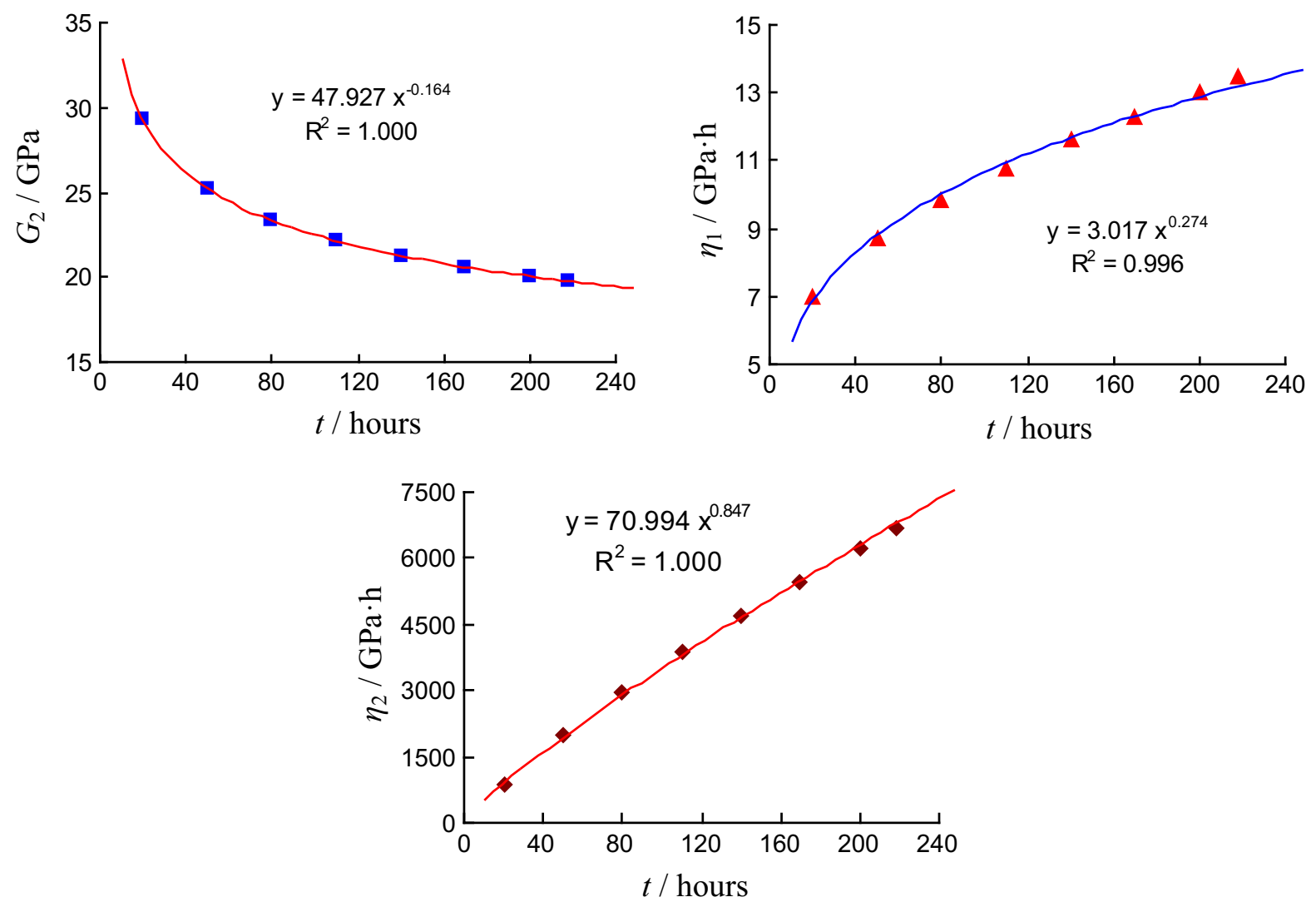

Fig. 3 Influence of time on creep parameters in Burgers visco-elastic triaxial creep model 
to predict accurately long-term creep deformation, which can be further illustrated by the following description.

The triaxial creep experimental data in 80 and $170 \mathrm{~h}$ are used to inverse the creep parameters on the basis of Burgers triaxial creep model, while the triaxial creep experimental data in 80-218 and 170-218 h are used to verify the predicted effect of Burgers triaxial creep model. Figure 4 a shows the comparison between Burgers triaxial creep model curves on basis of inversing parameters with the experimental data within time scale of $80 \mathrm{~h}$ and long-term visco-elastic triaxial creep deformation (80-218 h). Figure 4b depicts the comparison between Burgers triaxial creep model curves on basis of inversing parameters with the experimental data within time scale of $170 \mathrm{~h}$ and long-term visco-elastic triaxial creep deformation (170-218 h). From Fig. 4a, we can see that Burgers triaxial creep model is not very good for predicting visco-elastic creep behavior in $80-218 \mathrm{~h}$, and the predicted creep deformation is distinctly higher than the experimental value. However by comparing Fig. 4a with Fig. 4b, we can conclude that Burgers triaxial creep model curve on basis of inversing parameters within the larger time scale is in a better agreement with the experimental result.

\section{Nonlinear visco-elastic triaxial creep model}

From Fig. 4, it can be seen that Burgers visco-elastic triaxial creep model (Eq. 11) with five parameters is very difficult to describe accurately visco-elastic triaxial creep characteristics of coal. The main reason for this is that four components in Burgers viscoelastic creep model are all linear, which can not make a reasonable reflection for nonlinear visco-elastic creep behavior of coal. Taking into account that the Burgers visco-elastic triaxial creep model is not very good for predicting long-term deformation characteristics of coal specimen (Fig. 4), therefore here a modified nonlinear visco-elastic triaxial creep model is constructed by introducing a time index to better predict the long-term deformation behavior of coal than that by linear visco-elastic triaxial creep model. The nonlinear visco-elastic triaxial creep model can be shown as follows.

$$
\begin{aligned}
\varepsilon_{1}= & \frac{\sigma_{1}+2 \sigma_{3}}{9 K}+\frac{\sigma_{1}-\sigma_{3}}{3 G_{1}} \\
& +\frac{\sigma_{1}-\sigma_{3}}{3 G_{2}}\left[1-\exp \left(-\frac{G_{2}}{A}\left(\frac{t}{t_{0}}\right)^{n}\right)\right]
\end{aligned}
$$

where $t_{0}$ is a reference time, which is set to 1.0 (the units of $t_{0}$ are the same as those to $t$ ), $n$ is time index and $A$ is a creep parameter.

The five parameters in the above nonlinear triaxial creep model (Eq. 13) can be identified by the same method as presented in Burgers triaxial creep model. Table 2 lists nonlinear triaxial creep model parameters of coal with different time scales. From Table 2, it can be seen that the nonlinear triaxial creep model parameters are not distinctly dependent on time scale, which is different from that for Burgers triaxial creep model. The above analysis shows that nonlinear triaxial creep model parameters identified by short-term creep test results can be used to predict accurately long-term visco-elastic triaxial deformation. In order to understand this
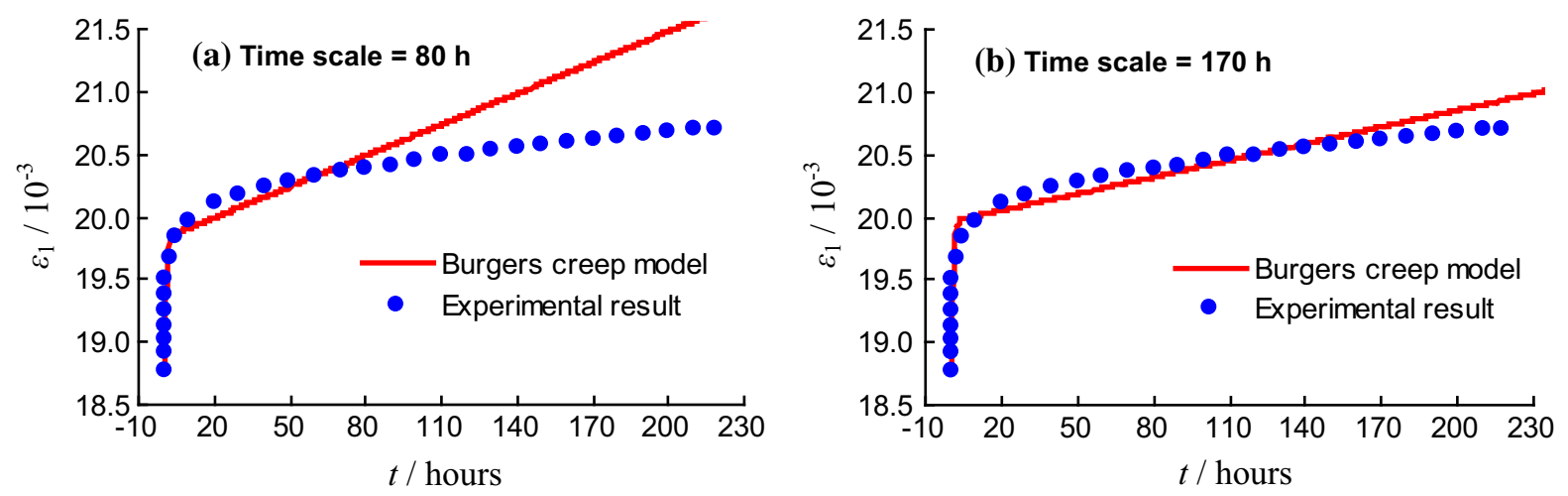

Fig. 4 Comparison between Burgers triaxial creep model curves on basis of inversing parameters with the experimental data within time scale of $80,170 \mathrm{~h}$ and long-term visco-elastic triaxial creep deformation 
Table 2 Nonlinear viscoelastic creep model parameters of coal with axial differential stress level of $73 \mathrm{MPa}\left(\sigma_{3}=30 \mathrm{MPa}\right)$

\begin{tabular}{llllllll}
\hline Time scale $(\mathrm{h})$ & $K(\mathrm{GPa})$ & $G_{1}(\mathrm{GPa})$ & $G_{2}(\mathrm{GPa})$ & $A(\mathrm{GPa})$ & $n$ & $R^{2}$ & $Q$ \\
\hline 20 & 4.131 & 1.690 & 17.74 & 22.31 & 0.431 & 0.995 & $1.11 \times 10^{-6}$ \\
50 & 4.131 & 1.690 & 15.73 & 24.49 & 0.386 & 0.996 & $1.32 \times 10^{-6}$ \\
80 & 4.131 & 1.690 & 14.46 & 25.85 & 0.357 & 0.996 & $1.55 \times 10^{-6}$ \\
110 & 4.131 & 1.690 & 13.41 & 26.91 & 0.334 & 0.995 & $1.77 \times 10^{-6}$ \\
140 & 4.131 & 1.690 & 12.39 & 27.84 & 0.314 & 0.995 & $2.00 \times 10^{-6}$ \\
170 & 4.131 & 1.690 & 11.43 & 28.65 & 0.298 & 0.994 & $2.15 \times 10^{-6}$ \\
200 & 4.131 & 1.690 & 10.54 & 29.35 & 0.284 & 0.994 & $2.24 \times 10^{-6}$ \\
218 & 4.131 & 1.690 & 10.05 & 29.71 & 0.277 & 0.994 & $2.23 \times 10^{-6}$ \\
Average & 4.131 & 1.690 & 13.22 & 26.89 & 0.340 & 0.995 & $1.80 \times 10^{-6}$ \\
\hline
\end{tabular}

point more deeply, Fig. 5a, b, respectively, show the comparison between nonlinear triaxial creep model curves on the basis of inversing parameters with the experimental data within time scale of $80,170 \mathrm{~h}$ and long-term visco-elastic triaxial creep deformation (80-218 h) and (170-218 h). From Fig. 5a, we can see that the predicted creep deformation by nonlinear triaxial creep model within time scale of $80 \mathrm{~h}$ is a little lower than the experimental value, while when time scale reaches $170 \mathrm{~h}$, the nonlinear triaxial creep model is much better for predicting viscoelastic creep behavior.

In order to further illustrate the dominance of the nonlinear triaxial creep model by comparing with Burgers triaxial creep model, Fig. 6 shows the comparison of $R^{2}$ and $Q$ identified by nonlinear and Burgers triaxial creep model with various time scales, respectively. From Fig. 6, it can be seen that $R^{2}$ for nonlinear triaxial creep model is higher than that for Burgers triaxial creep model. However, $Q$ for
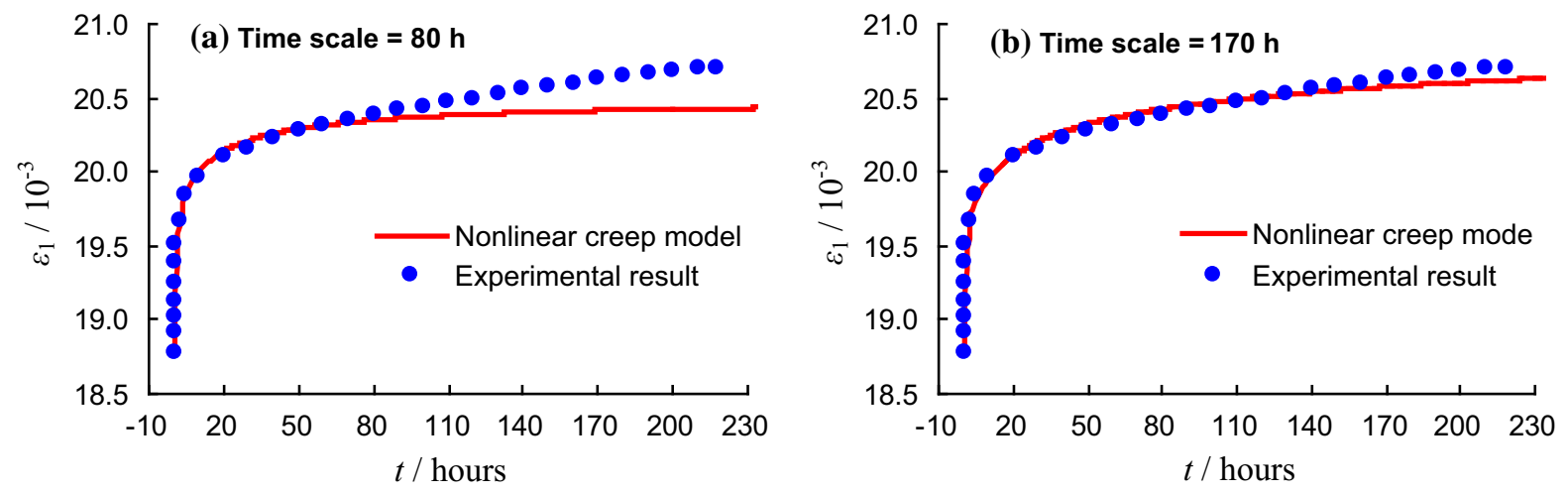

Fig. 5 Comparison between nonlinear triaxial creep model curves on basis of inversing parameters with the experimental data within time scale of $80,170 \mathrm{~h}$ and long-term visco-elastic triaxial creep deformation

Fig. 6 Comparison of $R^{2}$ and $Q$ identified by nonlinear and Burgers triaxial creep model, respectively
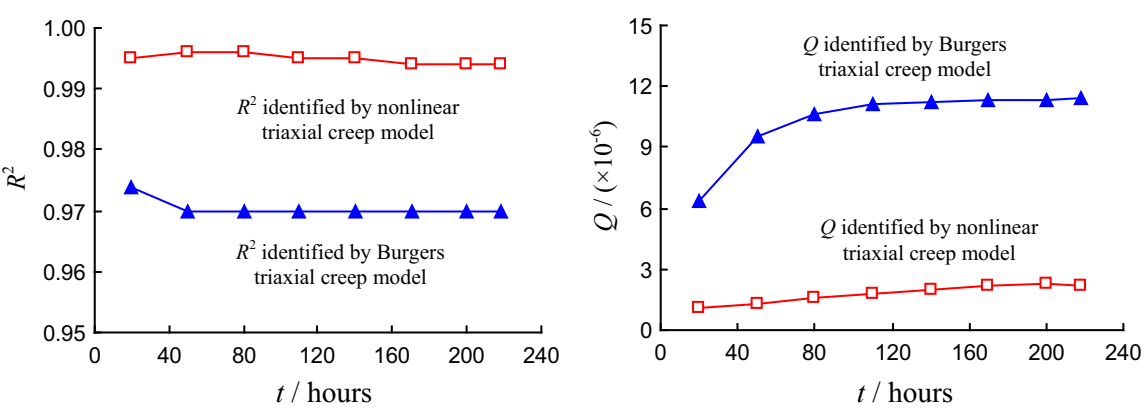


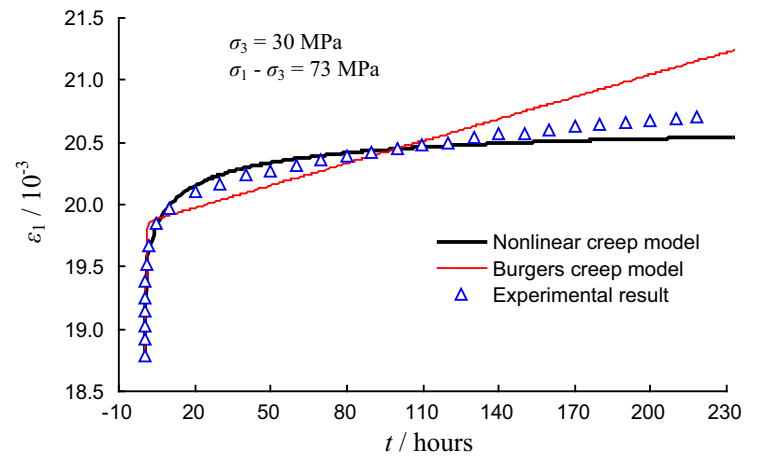

Fig. 7 Comparison between nonlinear, Burgers triaxial creep model (the creep parameters are the average value with various time scale) and experimental result

nonlinear triaxial creep model is smaller by one order than that obtained by Burgers triaxial creep model. Moreover, there are five parameters in the nonlinear triaxial creep model, which is the same as those in Burgers triaxial creep model. Besides, nonlinear triaxial creep model can predict very well for longterm triaxial deformation tendency of coal, therefore, nonlinear triaxial creep model of coal is better than Burgers triaxial creep model.

Figure 7 illustrates the comparison between nonlinear triaxial creep model and experimental result, and the creep parameters are the average value with various time scales, as listed in Table 2. At the same time, the comparison between Burgers triaxial creep model using the average creep parameters in Table 1 and experimental results is also plotted in Fig. 7. From Fig. 7, it can be seen clearly that nonlinear triaxial creep model using the average values as creep parameters has a good agreement with experimental result. However, Burgers triaxial creep model using the average values as creep parameters is not in a good agreement with experimental result, which is due to that Burgers triaxial creep model parameters has a certain time scale effect.

\section{Nonlinear accelerating creep model}

From Fig. 7, it can be seen that Burgers and nonlinear visco-elastic triaxial creep model can only describe the primary creep stage and steady-state creep stage, but can not describe the accelerating creep stage. Therefore, it is necessary to put forward a new nonlinear accelerating creep model in order to describe the complete creep curve of coal as shown in Fig. 2 b.
Up to now, there are mainly two methods to construct non-linear accelerating creep models of rock. One method is to replace conventional linear rheological components such as Hooker body, Newton body and Plastic body with nonlinear components (Bahar et al. 1995; Yang et al. 2007). The other method is to adopt new theory (such as fracture and damage theory etc.) to construct creep model of rock (Grgic et al. 2003; Enrico and Tsutomu 2001; Pellet et al. 2005; Fabre and Pellet 2006; Sterpi and Gioda 2009). The proposed rheological models by the above two methods can all describe the accelerating creep stage of rock material. In this research, the first method is adopted to construct a new nonlinear accelerating creep model of coal.

Heap et al. (2009) depicted an evolution process of spatial AE hypocenters during the complete triaxial creep test of Darley Dale sandstone. A total of $1877 \mathrm{AE}$ hypocenters were located during the experiment, of which 592 occurred during the primary creep phase, 702 during secondary creep and 583 during tertiary creep. The experimental result from Heap et al. (2009) showed that: (1) diffuse and distributed during primary creep, (2) distributed with some suggestion of localization or "mixed mode" during secondary creep and (3) increasingly localized along the eventual fault plane during tertiary creep. Therefore, the main reason that rock material produces nonlinear creep deformation can be described as follows. Due to the long-term action of a constant axial differential stress level, microscopic crack in the rock will undergo a process of propagation and fracture with the increase of time. So the nonlinear creep deformation of rock material is actually a function of time. By assuming that the nonlinear creep deformation of rock is the Weibull distribution function of time, a new nonlinear rheological component (Fig. 8a) is put forward, which can describe accurately the accelerating creep deformation of rock material. Figure $8 \mathrm{~b}$ shows the relation between the creep deformation at a constant stress level and time, and the creep equation under one-dimensional stress state is

$\varepsilon_{1}=\frac{\sigma}{E}\left[1-\exp \left(-\left(\frac{H\left(t-t_{2}\right)}{t_{c}-t_{2}}\right)^{\gamma}\right)\right]$

where $E$ is elastic modulus, $t_{2}$ is the time from steadystate creep stage to accelerating creep stage; $t_{\mathrm{c}}$ is the time of creep failure; $\gamma$ is time index. In Eq. (14), $H$ can be expressed as follows. 
(a)
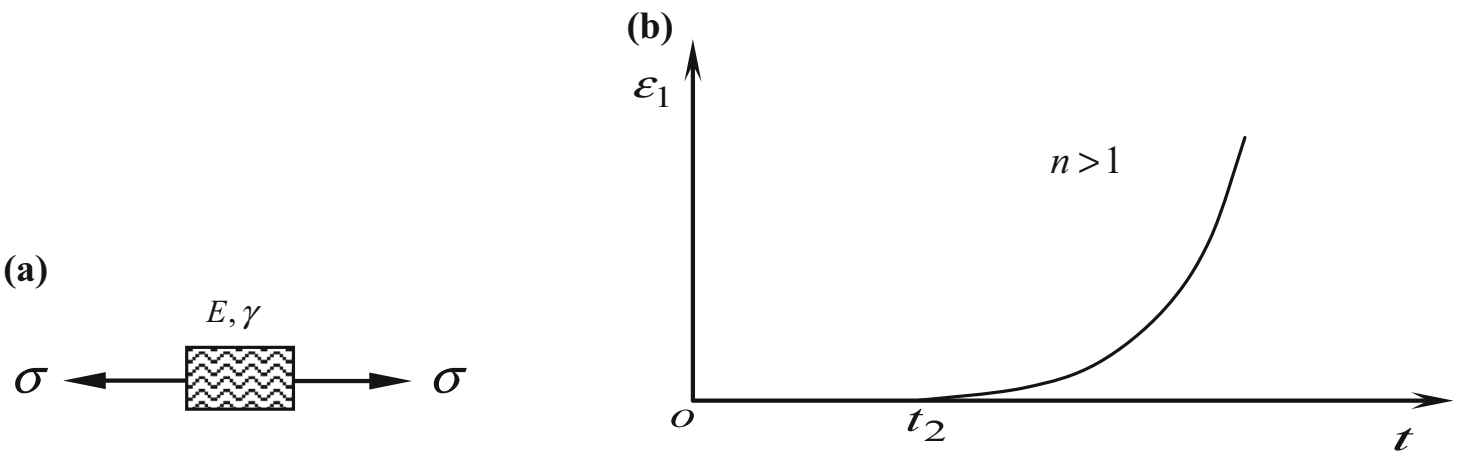

Fig. 8 Nonlinear rheological component and its creep curve. a Nonlinear rheological component, b Creep curve of nonlinear rheological component

$H\left(t-t_{2}\right)= \begin{cases}0, & t \leq t_{2} \\ t-t_{2}, & t>t_{2}\end{cases}$

In accordance with Eq. (14) and Fig. 8, it can be seen that with the increase of time, the axial strain and axial strain rate of rock take on a nonlinear increase; therefore, the nonlinear rheological component can reflect the behavior of nonlinear accelerating creep of rock. If the nonlinear rheological component and Burgers visco-elastic model are connected in series, a new nonlinear accelerating rheological model (Fig. 9) can be obtained.

The creep equation of nonlinear accelerating rheological model under one-dimensional stress state (i.e. uniaxial compression state) is

$$
\begin{aligned}
\varepsilon_{1}= & \frac{\sigma}{E_{1}}+\frac{\sigma}{E_{2}}\left[1-\exp \left(-\frac{E_{2}}{\eta_{1}} t\right)\right]+\frac{\sigma}{\eta_{2}} t \\
& +\frac{\sigma}{E_{3}}\left[1-\exp \left(-\left(\frac{H\left(t-t_{2}\right)}{t_{c}-t_{2}}\right)^{\gamma}\right)\right]
\end{aligned}
$$

In accordance with the same method as deducing Burgers visco-elastic triaxial creep model under threedimensional stress state, we also can obtain the nonlinear accelerating creep equation of rock under conventional triaxial compression stress condition $\left(\sigma_{2}=\sigma_{3}\right)$

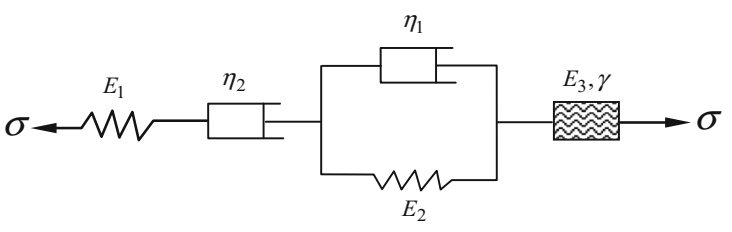

Fig. 9 Nonlinear accelerating rheological model

$$
\begin{aligned}
\varepsilon_{1}= & \frac{\sigma_{1}+2 \sigma_{3}}{9 K}+\frac{\sigma_{1}-\sigma_{3}}{3 G_{1}}+\frac{\sigma_{1}-\sigma_{3}}{3 G_{2}} \\
& {\left[1-\exp \left(-\frac{G_{2}}{\eta_{1}} t\right)\right]+\frac{\sigma_{1}-\sigma_{3}}{3 \eta_{2}} t } \\
& +\frac{\sigma_{1}-\sigma_{3}}{3 G_{3}}\left[1-\exp \left(-\left(\frac{H\left(t-t_{2}\right)}{t_{c}-t_{2}}\right)^{\gamma}\right)\right]
\end{aligned}
$$

The seven parameters in the above nonlinear accelerating creep model (Eq. 17) can be identified by the following method. First using the creep experimental data in time interval $\left(0, t_{2}\right)$, the five parameters $K, G_{1}, G_{2}, \eta_{1}$ and $\eta_{2}$ can be confirmed as the same method presented in Burgers triaxial creep model.

After confirming the five parameters $K, G_{1}, G_{2}, \eta_{1}$ and $\eta_{2}$, when $t=t_{\mathrm{c}}$ and $\varepsilon_{1}=\varepsilon_{\mathrm{c}}$, the following equation can be obtained.

$$
\begin{aligned}
\varepsilon_{c} & -\frac{\sigma_{1}+2 \sigma_{3}}{9 K}-\frac{\sigma_{1}-\sigma_{3}}{3 G_{1}} \\
& -\frac{\sigma_{1}-\sigma_{3}}{3 G_{2}}\left[1-\exp \left(-\frac{G_{2}}{\eta_{1}} t_{c}\right)\right]-\frac{\sigma_{1}-\sigma_{3}}{3 \eta_{2}} t_{c} \\
= & \frac{\sigma_{1}-\sigma_{3}}{3 G_{3}}\left(1-e^{-1}\right)
\end{aligned}
$$

In this research, according to Fig. 2, we can confirm that $t_{2}$ equals to $0.8306 \mathrm{~h}$ and $t_{\mathrm{c}}$ equals to 0.9556 h. Combined with Eq. (18), we can confirm that $G_{3}=32.05 \mathrm{GPa}$. And then by using a certain creep experimental data in time interval $\left(t_{2}, t_{\mathrm{c}}\right)$, we can obtain the creep parameter $\gamma$. Thus, we confirm seven parameters $K, G_{1}, G_{2}, \eta_{1}, \eta_{2}, G_{3}$ and $\gamma$ in nonlinear accelerating creep model, which are listed in Table 3. 


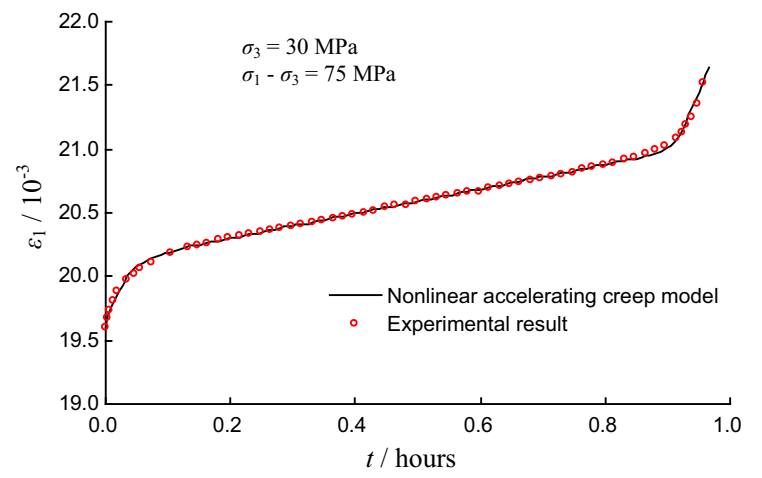

Fig. 10 Comparison between nonlinear accelerating creep model and experimental results of coal

Figure 10 shows the comparison between nonlinear accelerating creep model and experimental results of coal. From Fig. 10, it can be seen that the nonlinear accelerating creep model is in a good agreement with experimental result, which shows the rightness and reasonability of nonlinear accelerating creep model in this research.

\section{Conclusions}

(1) The tested coal shows good visco-elastic deformation behavior at differential stress of $73 \mathrm{MPa}$ and confining pressure of $30 \mathrm{MPa}$. Based on obtained visco-elastic triaxial creep experimental results of coal, Burgers visco-elastic triaxial creep model are firstly used to identify the creep parameters by $R^{2}$ and $Q$. Burgers triaxial creep model parameters have a strong time scale effect, which shows that the visco-elastic shear modulus $G_{2}$ of rock decreases but the visco coefficient $\eta_{1}$ and $\eta_{2}$ increases with increasing time.

(2) Taking into account that Burgers triaxial creep model is not very good for predicting long-term deformation behavior of coal, a nonlinear viscoelastic triaxial creep model is constructed by introducing a time index, which can predict better for long-term deformation behavior of coal than that by Burgers triaxial creep model. $R^{2}$ identified by nonlinear triaxial creep model is higher but $Q$ is smaller by one order than that by Burgers triaxial creep model. The predicted curve by nonlinear triaxial creep model agrees very well with experimental data, which shows the reasonability of nonlinear visco-elastic triaxial creep model.

(3) Burgers and nonlinear visco-elastic triaxial creep models can only describe the primary creep stage and steady-state creep stage, but can not describe the accelerating creep stage. Therefore in order to describe the complete creep curve of coal, a new nonlinear accelerating creep model is put forward, which is in a good agreement with experimental result.

Finally, it should be pointed out that this research only constructs the nonlinear visco-elastic and accelerating creep model for coal under conventional triaxial compression, which the reasonability of constructed nonlinear triaxial creep model is also testified. However, the creep mechanical of rock material is dependent to a lot of factors, such as the confining pressure, water pressure, temperature and humidity, etc. Therefore, in the future, we will carry out more triaxial creep tests for rock material and strengthen nonlinear triaxial creep model of rock material by taking into account more affected factors.

Table 3 Nonlinear accelerating creep model parameters of coal with axial differential stress level of $75 \mathrm{MPa}\left(\sigma_{3}=30 \mathrm{MPa}\right)$

\begin{tabular}{llllllll}
\hline Specimen & $K(\mathrm{GPa})$ & $G_{1}(\mathrm{GPa})$ & $G_{2}(\mathrm{GPa})$ & $\eta_{1}(\mathrm{GPa})$ & $\eta_{2}(\mathrm{GPa} \mathrm{h})$ & $G_{3}(\mathrm{GPa})$ & $\gamma$ \\
\hline Coal & 4.054 & 1.658 & 49.11 & 1.357 & 26.01 & 32.05 & 5.171 \\
\hline
\end{tabular}


Acknowledgments This research was financially supported by National Natural Science Foundation of China (Grant Nos. 41272344, 51323004) and Natural Science Foundation of Jiangsu Province for Distinguished Young Scholars (Grant No. BK20150005). The authors would also like to express their sincere gratitude to the editor and the anonymous reviewers for their valuable comments, which have greatly improved this paper.

\section{References}

ASTM D-4406 (1996) Test method for creep of cylindrical rock core specimen in triaxial compression. ASTM, USA

Bahar R, Cambou B, Fry JJ (1995) Forecast of creep settlements of heavy structures using pressure meter tests. Comput Geotech 17:507-521

Bonini MC, Debernardi D, Barla M, Barla G (2009) The mechanical behaviour of clay shales and implications on the design of tunnels. Rock Mech Rock Eng 42:361-388

Cao SG, Liu YB, Zhang LQ, Jiang YD (2007) Experimental on acoustic emission of outburst-hazardous coal under uniaxial compression and creep. J China Coal Soc 32(12):1264-1268 (in Chinese)

Enrico M, Tsutomu Y (2001) A non-associated viscoplastic model for the behavior of granite in triaxial compression. Mech Mater 33:283-293

Fabre G, Pellet F (2006) Creep and time-dependent damage in argillaceous rocks. Int J Rock Mech Min Sci 43(6): 950-960

Feng ZJ, Wan ZJ, Zhao YS, Li GW, Zhang Y, Wang C (2010) Experimental investigation into deformation characteristics of anthracite under thermo-mechanical coupling conditions. Chin J Rock Mech Eng 29(8):1624-1630 (in Chinese)

Grgic D, Homand F, Hoxha D (2003) A short- and long-term rheological model to understand the collapses of iron mines in Lorraine, France. Comput Geotech 30(7): 557-570

Heap MJ, Baud P, Meredith PG et al (2009) Time-dependent brittle creep in Darley Dale sandstone. J Geophys Res 114(B07203):1-22

Li YS, Yang YJ, Yang SQ, Ma ZG (2011) Deformation and acoustic emission behaviors of coal under triaxial compression and pore water pressure. J Univ Sci Technol Beijing 33(6):658-663 (in Chinese)

Medhurst TP, Brown ET (1998) A study of the mechanical behavior of coal for pillar design. Int J Rock Mech Min Sci 35(8):1087-1105

Pellet F, Hajdu A, Deleruyelle F, Besnus F (2005) A viscoplastic model including anisotropic damage for the time dependent behaviour of rock. Int J Numer Anal Methods Geomech 29:941-979

Sterpi D, Gioda G (2009) Visco-plastic behaviour around advancing tunnels in squeezing rock. Rock Mech Rock Eng 42:319-339

Wang WZ, Ying GZ, Wang DK, Qin H (2010) A visco-elastoplastic creep model of outburst prone coal under triaxial compression. J Chongqing Univ 33(1):99-103 (in Chinese)

Xie HP, Zhao XP, Liu JF, Zhang R, Xue DJ (2012) Influence of different mining layouts on the mechanical properties of coal. Int J Rock Mech Min Sci 22:749-755

Xu J, Peng SJ, Tao YQ, Yang HW (2009) Experimental analysis of influence of creep on permeability of gas-bering coal. Int J Rock Mech Min Sci 28(11):2273-2279 (in Chinese)

Yang SQ, Xu WY, Yang SL (2007) Investigation on shear rheological mechanical properties of shale in Longtan Hydropower Project. Rock Soil Mech 28(5):895-902 (in Chinese)

Yin GZ, Wang H, Zhang DM (2011) Creep experimental and theory model on coal containing gas under the condition of unloading confining pressure. J China Coal Soc 36(12):1963-1967 (in Chinese) 